\title{
Bemerkungen zu der Arbeit von H. Euler und A. Fodor: ¿Zur Kenntnis des Hefegummis».
}

Von

E. Salkowski.

(Der Redaktion zugegangen am 7. Juli 1911.)

Die Arbeit von Euler und Fodor in dieser Zeitschrift (Bd. 72, S. 340) nötigt mich $\mathrm{zu}$ einigen Bemerkungen.

I. Euler und Fodor beginnen ihre Abhandlung mit den Worten:

«Stellt man Präparate von Hefeinvertase dar, sei es durch Extraktion der getrockneten Zellen mit Wasser, sei es aus autolysierter Hefe, so enthält das enzymatisch wirksame Produkt reichliche Mengen eines Kohlenhydrates, welches Salkowski als Hefegummi bezeichnet hat. Die Invertasepräparate liefern nämlich mit Fehlingscher Lösung das von Salkowski beschriebene Kupfersalz des Gummis, das nach der Hydrolyse Mannosehydrazon liefert.»

Danach könnte es scheinen, als ob Euler und Fodor den Gehalt der Invertinpräparate an Hefegummi erst entdeckt hätten, während dieser Befund tatsächlich von mir herrührt: ${ }^{1}$ ) ich habe nicht allein auf den Gehalt der Invertinpräparate an Hefegummi, welcher eine Aufklärung für den niedrigen $\mathrm{N}$-Gehalt der Invertinpräparate gibt, und auf den Nachweis durch Fehlingsche Lösung aufmerksam gemacht, sondern auch in einer Anzahl von Invertinpräparaten den Gehalt an Hefegummi quantitativ bestimmt. Den Autoren scheint dies entgangen $z u$ sein, sonst könnten sie den Sachverhalt doch nicht als scheinbar neu mitteilen, ohne sich auf mich $\mathrm{zu}$ beziehen oder abekanntlich, hinzuzusetzen.

II. Weiterhin sagen die Verfasser:

«Nun haben es einerseits die im hiesigen Laboratorium angestellten Reinigungsversuche sehr unwahrscheinlich gemacht, daß die Invertase, wie früher fast durchweg angenommen wurde und jetzt noch vielfach angenommen wird, ein Einweißkörper ist, denn nach der Behandlung der Präparate mit Kaolin und anderen Adsorptionsmitteln für Eiweißstoffe bleibt ein Rest von hoher Aktivität zurück. Anderseits sind offenbar eine Reihe chemischer Reaktionen dem Hefegummi und der Invertase gemeinsam, und es wird hierdurch die Annahme nahegelegt, daß die Invertase selbst ein höheres Kohlenhydrat und zum Hefegummi chemisch verwandt ist.»

1) Diese Zeitschrift, Bd. 31, S. 305 (1900/1901). 
Dazu habe ich folgendes zu bemerken:

1. $\mathrm{Ob}$ das Verhalten $\mathrm{zu}$ Kaolin ganz beweisend ist, ${ }^{1}$ ) mag hier unerörtert bleiben, jedenfalls hat schon vor langen Jahren M. Barth, ${ }^{2}$ ) der unter meiner Leitung arbeitete, mit aller Bestimmtheit ausgesprochen, daß das Invertin kein Eiweißkörper sei, ebenso ich selbst später auf Grund von Reaktionen (1. c., S. 315).

2. Welche Reaktionen dem Invertin und dem Hefegummi gemeinsam seien, haben die Autoren leider nicht näher ausgeführt. Eine Beziehung des Invertins zu Kohlenhydraten ist schon von verschiedenen Autoren, so von Osborne und Kölle, dann auch von Hafner angenommen worden, in der zitierten Arbeit, sowie in einer späteren, auf die ich gleich zu sprechen komme, habe ich die Gründe erörtert, warum i ch, im Gegensatz zu den genannten Autoren, in dem Gehalt der Invertinpräparate an Gummi nur eine Verunreinigung sehe. Aber ganz abgesehen von den Gründen, die für meine Auffassung sprechen, ist es mir ja gelungen (nicht konstant, aber doch wiederholt), sehr wirksame Invertinlösungen herzustellen, die keine Spur von Gummi enthielten. ${ }^{3}$ ) Ich verstehe nicht, wie man danach die Frage eines Zusammenhanges des Invertins mit dem Kohlenhydrat überhaupt noch aufwerfen oder gar eine Zusammengehörigkeit annehmen kann, es sei denn, daß den Autoren meine Mitteilung entgangen ist, was wenig Wahrscheinlichkeit für sich hat. Was das Invertin ist, kann ich freilich nicht sagen; wenn ich aber ausnahmsweise eine Vermutung aussprechen darf - das ist ja im allgemeinen nicht üblich, vielleicht aber in diesem Fall durch besondere Verhältnisse gerechtfertigt - , so geht diese dahin, daß das Invertin vielleicht das Magnesiumsalz einer stickstoff- und phosphorhaltigen Säure ist. Es spricht sehr vieles dafür; ich gedenke noch darauf zurückzukornmen.

III. Auf Seite 341 sagen die Verfasser:

-Weit besser erwies sich die von Salkowski angegebene Darstellung aus autolysierter Hefe, insofern dabei ein reineres Produkt erhalten wurde.s

Dieser Satz könnte zu der mißverständlichen Annahme führen, daß ich autolysierte Hefe als Ausgangsmaterial benutzt oder empfohlen habe. Das ist nie geschehen. ${ }^{4}$ ) Ich habe nur die Darstellung aus Preßhefe ${ }^{5}$ ) beschrieben. Sie liefert, wenn man genau nach meiner Vorschrift

1) Die Angabe rührt übrigens von L. Michaelis (vgl. Biochem. Zeitschr., Bd. 7, S. 488) her; nach ihm besitzt die Invertinlösung nach Behandlung mit Kaolin sogar ihre volle invertierende Wirkung.

₹) Ber. d. deutsch. chem. Gesellsch., Bd. 11, S. 474 (1878).

${ }^{3}$ ) Diese Zeitschrift, Bd. 61, S. 124. Das ist mir seitdem noch öfters geglückt.

4) Die Verfasser wollen wohl sagen: das von Salkowski angegebene Verfahren, auf autolysierte Hefe angewendet».

5) Ber. d. deutsch. chem. Gesellsch., Bd. 27, S. 497 (1894). 
arbeitet, ein absolut reines Produkt, ich habe dieser Vorschrift nichts hinzuzusetzen. Es ist mir nicht verständlich, warum die Verfasser dieses äußerst einfache Verfahren nicht angewendet haben, umsoweniger, als Meigen und Spreng ${ }^{1}$ ) damit zu guten Resultaten gelangt sind. Die Darstellung aus dem «durch mehrwöchentliche Autolyse aus Brauereihefe erhaltenen Safts erscheint mir recht unzweckmäßig, da dieser wohl überhaupt nur wenig Hefegummi enthält. Selbstverständlich tritt bei meiner Darstellung auch nicht die von den Verfassern beobachtete Reduktion beim Erhitzen mit Fehlingscher Lösung ein.

4. Schließlich noch eine Bemerkung, die in keiner direkten Beziehung zu der Publikation von Euler und Fodor steht. Es ist in neuerer Zeit Mode geworden, das Invertin sInvertase zu nennen. Diese Benennung halte ich für durchaus verfehlt. Es ist gewiß nichts dagegen zu sagen, wenn man die Namen der Fermente auf die Endigung sase ausgehen läßt und sie von dem Substrat ableitet, auf welches das Ferment einwirkt, also von Maltase, Protease, Nuclease spricht, dann muß man aber auch das Prinzip wahren und das Invertin als Saccharase bezeichnen. Dagegen ist nichts einzuwenden, die Bezeichnung aInvertin. aber in sInvertase» umzuändern, dazu liegt nicht der geringste Grund vor.

1) Diese Zeitschrift, Bd. 55̃, S. 48 (1908). 